OPEN ACCESS

Edited by:

Jiajin Yuan,

Southwest University, China

Reviewed by:

Yixue Lou,

Shenzhen University, China

Qiwei Yang,

Chengdu Medical College, China

${ }^{*}$ Correspondence:

Jie Chen

xIxchen@163.com

Specialty section:

This article was submitted to Emotion Regulation and Processing, a section of the journal Frontiers in Behavioral

Neuroscience

Received: 16 January 2020 Accepted: 18 March 2020

Published: 07 May 2020

Citation:

Xiao R, Liu C, Chen J and Chen J (2020) The Influence of Music Tempo on Inhibitory Control: An ERP Study.

Front. Behav. Neurosci. 14:48.

doi: 10.3389/fnbeh.2020.00048

\section{The Influence of Music Tempo on Inhibitory Control: An ERP Study}

\author{
Rong Xiao ${ }^{1,2}$, Cuihong Liu ${ }^{1,2}$, Jiejia Chen ${ }^{1,2}$ and Jie Chen ${ }^{1,2 *}$ \\ ${ }^{1}$ School of Educational Science, Hunan Normal University, Changsha, China, ${ }^{2}$ Cognition and Human Behavior Key \\ Laboratory of Hunan Province, Hunan Normal University, Changsha, China
}

The purpose of the present study is to investigate the influence of music tempo on inhibition control. An electroencephalogram (EEG) was recorded when participants performed a Go/No-go task while listening to slow (54 bpm), medium-paced (104 bpm), fast (154 bpm), or no music. The behavioral results showed that the accuracies for the No-go trials were lower in the fast than in the slow tempo music conditions, while the accuracies for the Go trials were also lower in the fast tempo than in no music conditions. The event-related potential (ERP) study results showed that larger N2 and P3 amplitudes were elicited by No-go than by Go conditions. Moreover, the difference N2 (N2d) amplitudes observed by No-go vs. Go condition were larger in fast music than in medium-paced, slow, and no music conditions, indicating more consumption of cognitive resources in the process of conflict monitoring under the fast music condition. However, no such differences were observed among medium-paced, slow, and no music conditions. In addition, the difference P3 (P3d) amplitudes, an index of response inhibition, were not significant among these four music conditions. The present study showed a detrimental influence of music tempo on inhibition control. More specifically, listening to fast music might impair an individual's ability to monitor conflict when performing the inhibitory control task.

Keywords: music tempo, inhibitory control, Go/No-go paradigm, N2, P3

\section{INTRODUCTION}

The popularity of music in the field of psychological research has been increasing. More and more researchers regard music as the product of a general-purpose cognitive architecture and then discuss it from different perspectives of musical elements (e.g., mode, rhythm, tempo, etc.; Sutton and Lowis, 2008; Levitin et al., 2018; Navarro et al., 2018). An investigation into these elements of music not only has strong operability and practical significance but also is the basis for our understanding of the effects of music on human cognition.

Music tempo, which is measured in terms of beats per minute (bpm), is a representative of the basic dimension of music (Karageorghis et al., 2011). Moreover, it has been found that the tempo of music can affect not only human's cognition such as attention, time perception, decision-making (North et al., 1998; Amezcua et al., 2005; Day et al., 2009), but also human's consumption, diet, or driving behaviors. For example, it was found that participants made faster stimulus evaluation and response in fast than in slow tempo music conditions during a visual selective attention task (Amezcua et al., 2005). The decision accuracy was also higher in fast than in slow tempo music conditions during a multi-attribute decision-making task (Day et al., 2009). Moreover, previous studies have shown that the in-store traffic in a supermarket 
could be speeded up and the daily gross sales volume increased when the background music played in fast tempo relative to that played in slow tempo (Milliman, 1982). Furthermore, the background music of fast tempo could shorten restaurant patrons' dining time (Milliman, 1986), with drinking speed increased (McElrea and Standing, 1992). Brodsky (2002) investigation into the impact of music tempo on simulated driving performance and vehicle control showed that the fast-paced music would increase the simulated driving speed and perceived speed estimate. Moreover, vehicular collision, lane crossings, and disregarded red traffic lights were more frequent during simulated driving in fast-paced than in low-paced background music conditions. Brodsky (2002) suggested that fast music could consume a driver's attentional resources and impaired their motor control.

Actually, most of our daily activities, such as consumption, shopping, diet, or driving behaviors as mentioned above, are associated with human's executive functions (also called cognitive control; Burkhard et al., 2018). Moreover, previous studies have demonstrated a close relationship between executive functions and musical training (Zuk et al., 2014). However, much less is known about the influence of music tempo on executive functions. Given the considerations mentioned above, the present study aimed to investigate the influences of music speed on executive functions. More specifically, we adopted event-related potentials (ERPs) and Go/No-go paradigm to investigate the temporal features underlying the influences of music speed on inhibition control. As an important subcomponent of executive functions, inhibition control is the ability to suppress inappropriate thoughts and responses (Diamond, 2013). Inhibitory control is frequently measured by using the Go/No-go paradigm, in which subjects were asked to respond to the "Go" stimulus and withhold their responses to the "No-go" stimulus (Falkenstein et al., 1999; Luijten et al., 2011).

Thus, in the present study, an electroencephalogram (EEG) was recorded when the participants performed the Go/No-go task while listening to slow (54 bpm), medium-paced (104 bpm), fast (154 bpm), or no music. Moreover, we put our focus on two ERP components, N2 and P3, both of which have been widely observed in the Go/No-go task. Specifically, the N2 amplitudes were larger for No-go trials relative to Go trials, reflecting the process of conflict monitoring (Nieuwenhuis et al., 2003). Moreover, the P3 amplitudes were also larger for No-go trials relative to Go trials, indexing the process of response inhibition (Falkenstein et al., 1999). In order to highlight the No-go N2 and the No-go P3 effects, the difference $\mathrm{N} 2$ (N2d) and P3 (P3d) waveforms were observed by subtracting the Go from the No-go conditions (Falkenstein et al., 1999; Gajewski and Falkenstein, 2013; Burkhard et al., 2018). Thus, we aimed to explore whether or not music tempo could affect the inhibitory control as evidenced by behavioral and neural indices. If music tempo influenced the inhibitory control, then different Go and No-go accuracies as well as the N2d and P3d amplitudes would be expected among music of different tempos. Otherwise, no behavioral and neural differences would be observed.

\section{MATERIALS AND METHODS}

\section{Participants}

To establish the sample size, a priori statistical power analysis for a repeated-measures design was conducted using $G^{*}$ Power 3.1.9.2 (Faul et al., 2007). According to the software, a total sample size of $n=19$ would be required to obtain a medium effect size of Cohen's $f=0.25$ ( $a=0.05$, power $=0.8$; Cohen, 1988). To ensure a sufficient number of participants, a sample size of 26 participants $(10$ females, mean age $=19.5$ years, $\mathrm{SD}=1.4$ ) were selected in the present study. All subjects were right-handed, with normal or corrected-to-normal visual acuity and no history of neurological diseases or color blindness. Ethical approval for the study was obtained from the Research Ethics Committee of the Hunan Normal University. The participants also signed an informed consent form before the experiment and were given appropriate rewards upon completion of the experiment.

\section{Materials}

The first movement of Beethoven's "Moonlight Sonata" was selected at the original $54 \mathrm{bmp}$ for slow tempo musical excerpt. Similar to the previous studies (Brodsky, 2002; Bishop et al., 2014), this original musical excerpt was recomposed to $104 \mathrm{bmp}$ for the medium and $154 \mathrm{bmp}$ for the fast musical excerpts using the Adobe Audition CS6 (Adobe Systems Inc., San Jose, CA, USA) software. All the participants in this experiment are not familiar with these three musical excerpts. Dynamic earphones (Air Pods 2) with noise cancellation function were used for the participants to listen to the music. In addition to these three music conditions, there is also a no music condition, in which the participants performed the Go/No-go task with no audio input. The music loudness value is set to $70 \mathrm{~dB}$ SPL, which could be adjusted by the subjects at will to ensure maximum comfort.

\section{Procedure}

This study adopted the Go/No-go paradigm, which is a classical paradigm to investigate inhibition control (Diamond, 2013). The stimuli in this task were two kinds of shapes with different colors: a white rectangle, a purple rectangle, a white triangle, and a purple triangle. All the white stimuli were Go trials (75\%) and all the purple stimuli were No-go trials (25\%), with each type of stimulus presented randomly. Each trial was initiated by a small black cross presented for a duration ranging from 500 to $1,000 \mathrm{~ms}$. Afterwards, one of the four types of stimuli was presented for $500 \mathrm{~ms}$, which then was followed by a gray screen presented for $800 \mathrm{~ms}$ (see Figure 1). The participants were required to press a key on Go trials and not to press a key on No-go trials while listening to slow tempo, medium tempo, fast tempo, and no music. Thus, the present study included four blocks (the slow tempo, medium tempo, fast tempo, and no music blocks). Each block contained 240 trials (180 Go and 60 No-go trials), and the order of these four blocks was balanced across the participants. At the end of each block, a self-reported rate of this music was required on a scale of 1-9 in terms of induced pleasure (unpleasant to pleasant), arousal (calm to intense), and preference (dislike to like). After the rating, there was also a break of at least $5 \mathrm{~min}$. 


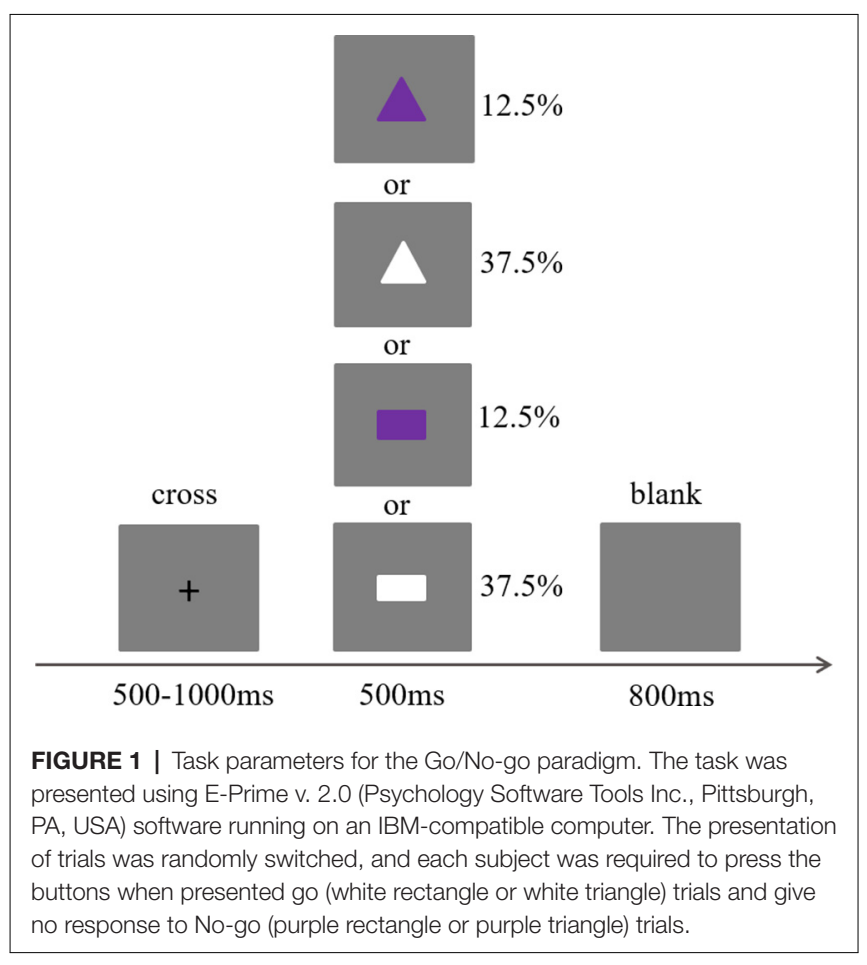

\section{Data Recording and Processing}

An EEG was recorded from 64 scalp sites using tin electrodes mounted in an elastic cap (Neuro Scan Inc.,) with an online reference to the $\mathrm{CPz}$. During the offline analysis, the EEG was re-referenced to the average of the right and the left mastoids. All interelectrode impedances were maintained under $5 \mathrm{~K} \Omega$. The EEG signals were amplified with a $0.1-30-\mathrm{Hz}$ bandpass filter and were continuously sampled at $500 \mathrm{~Hz} /$ channel. The EEG was averaged in $800 \mathrm{~ms}$ epochs (200-ms baseline) that were time-locked to the presentation of the stimulus mark. According to previous ERP literatures regarding the Go/Nogo task (Huster et al., 2010) and through the inspection of the topographic maps and grand-averaged ERP waveforms, we analyzed two specific components, N2 $(260-320 \mathrm{~ms})$ and P3 (400-500 ms) with the following regions: frontal (F3, $\mathrm{F} 1, \mathrm{Fz}, \mathrm{F} 2$, and $\mathrm{F} 4$ ), fronto-central (FC3, FC1, FCz, FC2, and FC4), central (C3, C1, Cz, C2, and C4), centro-parietal (CP3, $\mathrm{CP} 1, \mathrm{CPz}, \mathrm{CP} 2$, and $\mathrm{CP} 4)$, and parietal (P3, P1, $\mathrm{Pz}, \mathrm{P} 2$, and $\mathrm{P} 4)$ regions. A three-way repeated analysis of variance (ANOVA) was conducted on the mean amplitudes of $\mathrm{N} 2$ and P3, with music tempo (four levels: $54 \mathrm{bpm}, 104 \mathrm{bpm}$, $154 \mathrm{bpm}$, and no music), stimulus type (Go and No-go trials), and brain regions (five levels: frontal, fronto-central, central, centro-parietal, and parietal) as within-subject factors. The difference N2 and P3 waveforms were observed by subtracting the Go from the No-go conditions. In addition, one-way ANOVA was conducted on the behavioral accuracy and reaction times (RTs) with music tempo as within-subject factor. The degrees of freedom of the F-ratio were corrected by Greenhouse-Geisser. False discovery rate correction was applied for post hoc multiple comparisons.

\section{RESULTS}

\section{Behavioral Results}

The ANOVA for No-go accuracy showed a significant main effect of music tempo $\left(F_{(3,75)}=4.48, p=0.017, \eta_{\mathrm{p}}^{2}=0.15\right)$. The post hoc multiple comparisons revealed that the accuracies were lower in fast than in slow music conditions. The ANOVA for Go accuracy $\left(F_{(3,75)}=8.93, p=0.001, \eta_{\mathrm{p}}^{2}=0.26\right)$ and RT $\left(F_{(3,75)}=42.43, p<0.001, \eta_{\mathrm{p}}^{2}=0.71\right)$ also showed a significant main effect of music tempo. The accuracy in the no music condition was higher than those in the fast-paced $(p=0.006)$, the medium-paced $(p<0.001)$, and the slow-paced $(p=0.006)$ music conditions. RTs in the fast-paced music condition were shorter than those in the medium-paced and in the slow-paced music conditions ( $p s<0.004)$, in which RTs were also shorter than those in the no music condition ( $p$ s $<0.001$; Table 1).

In addition, the ratings on music-induced pleasure, arousal, and preference showed no significant main effects on arousal $\left(F_{(2,50)}=1.78, p=0.18, \eta_{\mathrm{p}}^{2}=0.07\right)$ and preference $\left(F_{(2,50)}=2.34\right.$, $\left.p=0.11, \eta_{\mathrm{p}}^{2}=0.086\right)$. A significant main effect on pleasure was observed $\left(F_{(2,50)}=5.93, p=0.005, \eta_{\mathrm{p}}^{2}=0.19\right)$, with higher scores for medium than for slow tempo musical excerpts $(p=0.006)$. However, no significant differences were observed between medium and fast $(p=0.19)$ or slow and fast $(p=0.08)$ tempo musical excerpts.

\section{ERP Results}

The ANOVA for $\mathrm{N} 2$ amplitudes showed a significant main effect on stimulus type $\left(F_{(1,25)}=50.35, p<0.001, \eta_{\mathrm{p}}^{2}=0.67\right)$, and the No-go condition elicited more negative $\mathrm{N} 2$ than the Go condition (see Figure 2A). Moreover, the interaction between stimulus type and music tempo was significant $\left(F_{(3,75)}=4.95, p=0.005\right.$, $\left.\eta_{\mathrm{p}}^{2}=0.17\right)$. The difference $\mathrm{N} 2$ amplitudes, obtained by subtracting the Go from the No-go conditions, were larger in the fast-paced music condition $(-5.19 \mu \mathrm{V})$ than those in the medium-paced $(-3.48 \mu \mathrm{V}, p=0.05)$, slow-paced $(-2.51 \mu \mathrm{V}, p<0.001)$, and no music $(-3.16 \mu \mathrm{V}, p=0.045)$ conditions. However, no significant differences were observed among the mediumpaced, slow-paced, and no music conditions ( $p s>0.26)$.

TABLE 1 | Results of the one-way repeated-measures analysis of variance (ANOVA) for the accuracy of Go and No-go trials and the reaction time (RT) of Go trials.

\begin{tabular}{lcccc}
\hline Conditions & $\begin{array}{c}\text { Fast (154 bpm) } \\
\text { Mean (SD) }\end{array}$ & $\begin{array}{c}\text { Medium-paced (104 bpm) } \\
\text { Mean (SD) }\end{array}$ & $\begin{array}{c}\text { Slow (54 bpm) } \\
\text { Mean (SD) }\end{array}$ & $\begin{array}{c}\text { No music } \\
\text { Mean (SD) }\end{array}$ \\
\hline Accuracy of No-go trials (\%) & $93.78(0.06)$ & $95.32(0.03)$ & $96.35(0.03)$ & $96.28(0.04)$ \\
Accuracy of Go trials (\%) & $94.64(0.08)$ & $95.86(0.04)$ & $95.23(0.07)$ & $99.25(0.02)$ \\
RT to Go trials in ms & $333.10(24.67)$ & $340.76(23.24)$ & $346.91(25.85)$ & $376.10(38.19)$ \\
\hline
\end{tabular}

Notes: $* p<0.05 ; * * p<0.01 ; * * *<0.001$. 


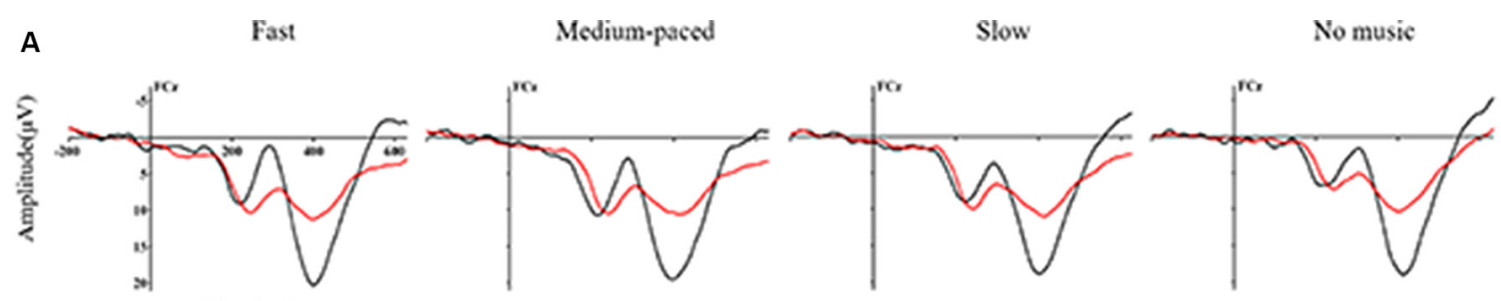

Time(ms)
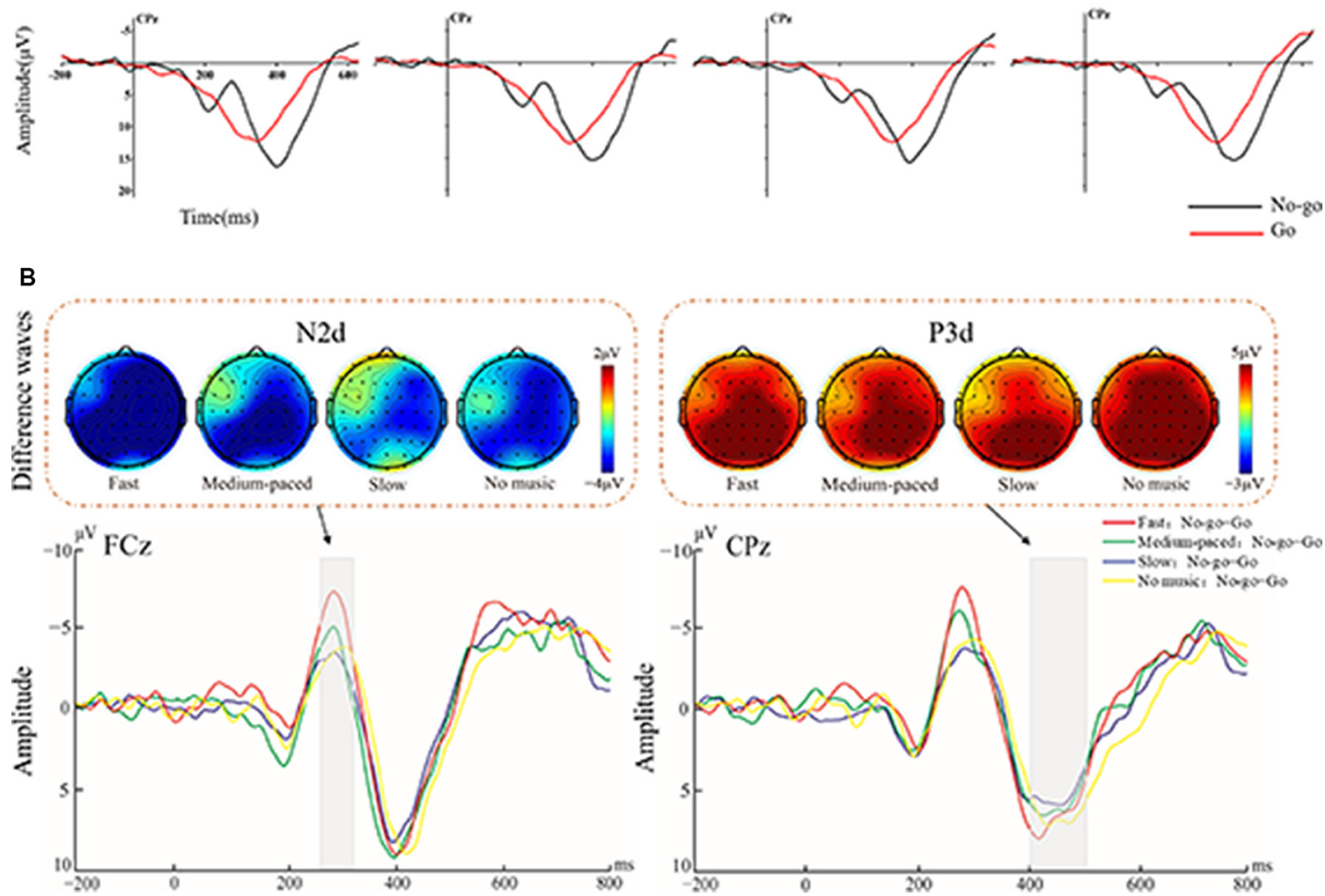

FIGURE 2 | (A) Averaged event-related potentials (ERPs) at electrode FCz and CPz for Go (red lines) and No-go (black lines) trials separated by conditions of music tempo (fast, medium-paced, slow, and no music). (B) The different waves (No-go minus Go) at FCz and CPz for the different tempos are shown in the lower panel. The amplitudes of the N2d and P3d components for tempos are indicated by different line colors: fast, red lines; medium-paced, green lines; slow, blue lines; and no music, yellow lines. The topographical maps of the N2d (left panel) and P3d (right panel) components for different tempos are shown in the upper panel.

The interaction between stimulus type and brain region was also significant $\left(F_{(4,100)}=4.88, p=0.02, \eta_{\mathrm{p}}^{2}=0.16\right)$. The $\mathrm{N} 2 \mathrm{~d}$ amplitudes were largest at the centro-parietal region. In addition, there was no significant interaction effect among music tempo, stimulus type, and regions $\left(F_{(12,300)}=1.88\right.$, $\left.p=0.13, \eta_{\mathrm{p}}^{2}=0.07\right)$.

The ANOVA for P3 amplitudes showed a significant main effect on stimulus type $\left(F_{(1,25)}=54.14, p<0.001, \eta_{\mathrm{p}}^{2}=0.68\right)$, and the No-go condition elicited larger P3 amplitudes than the Go condition (see Figure 2B). The interaction between stimulus type and brain region was significant $\left(F_{(4,100)}=7.13, p=0.008\right.$, $\left.\eta_{\mathrm{p}}^{2}=0.22\right)$. The P3d amplitudes were largest at the parietal region. However, no significant interaction effects were observed between stimulus type and music tempo $\left(F_{(3,75)}=1.005, p=0.39\right.$, $\left.\eta_{\mathrm{p}}^{2}=0.04\right)$ and among stimulus type, music tempo, and brain region $\left(F_{(12,300)}=1.53, p=0.2, \eta_{\mathrm{p}}^{2}=0.06\right)$.

\section{DISCUSSION}

The current study examined the influences of different tempos of music on inhibitory control by using the Go/No-go paradigm. The behavioral results showed that the accuracies for No-go trials were lower in the fast than in slow tempo music conditions, while the accuracies for Go trials were also lower in the fast tempo than in no music conditions. These behavioral results might indicate an impaired inhibitory control when listening to fast tempo music.

Consistent with previous studies (Nieuwenhuis et al., 2003), the present study showed larger N2 amplitudes in No-go than in Go conditions, irrespective of the type of background music. Moreover, we also observed a significant interaction effect between stimulus type and music tempo. The N2d amplitudes, obtained by subtracting the Go from the No-go conditions, 
were larger in the fast tempo music condition than in the three other conditions. The N2 component in the inhibitory control tasks was suggested to reflect the detection of response conflict (Nieuwenhuis et al., 2003) and also a recruitment of attentional resource for the following response inhibition (Van Veen and Carter, 2002; Yuan et al., 2012). Jodo and Kayama (1992) found that the No-go N2 amplitudes were larger under high than under low time pressure condition. The participants in the high time pressure condition were required to make Go responses within a shorter period, which thus resulted in fast responses to Go trials. Jodo and Kayama (1992) suggested that the faster responses to the Go trials could enhance the Go responses, which would be more difficult to be withheld on the appearance of the No-go trials. Thus, increased efforts were required to inhibit the Go response to No-go trials, which thus contributed to enhanced N2 amplitudes (Jodo and Kayama, 1992). In the current study, the behavioral responses to Go trials were faster in the fast tempo music condition than those in the three other conditions. This result was consistent with the previous study showing that faster responses were induced by listening to fast than to slow tempo music during a visual selective attention task (Amezcua et al., 2005). Thus, more cognitive efforts would be required to produce appropriate No-go response in a fast tempo music condition, which contributed to larger N2d amplitudes.

Moreover, consistent with previous studies (Falkenstein et al., 1999; Gajewski and Falkenstein, 2013), larger P3 amplitudes were observed for No-go trials relative to Go trials in the present study. It has been generally considered that the $\mathrm{P} 3$ predominantly represents motor or response inhibition (Enriquez-Geppert et al., 2010). However, we did not observe the interaction effect between stimulus type and music tempo. In other words, the P3d amplitudes were similar among the four music conditions. This finding suggested that the tempo of music did not affect the later response inhibition.

However, it should be noted that the tempo of the music is one of the potential factors for inducing emotion (Kim et al., 2018). Thus, the emotion effect induced by music tempo cannot be completely ruled out when investigating the influence of music tempo on inhibitory control and thus would form a contamination for the present study. However, the self-reported rate of music in terms of induced pleasure, arousal, and preference could rule out this possibility because there were no significant differences on arousal and preference rating among

\section{REFERENCES}

Amezcua, C., Guevara, M. A., and Ramos-Loyo, J. (2005). Effects of musical tempi on visual attention ERPs. Int. J. Neurosci. 115, 193-206. doi: 10.1080/00207450590519094

Bishop, D. T., Wright, M. J., and Karageorghis, C. I. (2014). Tempo and intensity of pre-task music modulate neural activity during reactive task performance. Psychol. Music 42, 714-727. doi: 10.1177/0305735613490595

Brodsky, W. (2002). The effects of music tempo on simulated driving performance and vehicular control. Transport. Res. F Traf. 4, 219-241. doi: 10.1016/s13698478(01)00025-0

Burkhard, A., Elmer, S., Kara, D., Brauchli, C., and Jäncke, L. (2018). The effect of background music on inhibitory functions: an ERP study. Front. Hum. Neurosci 12:293. doi: 10.3389/fnhum.2018.00293 these three types of music conditions. Although a significant main effect on pleasure was observed, no significant differences were observed between medium and fast or between slow and fast tempo music conditions. Thus, the ERP effects at the N2d were more likely specific to the tempo of music rather than the induced pleasure, arousal, and preference.

Taken together, the present study, using ERPs, demonstrated an obvious effect of music tempo on inhibition control. More specifically, listening to fast music would impair an individual's ability to monitor conflict. To our knowledge, this is the first time that the influences of music tempo on inhibitory control are directly investigated. In the future, the present findings should be replicated and verified by other experimental paradigms, especially the two-choice oddball task, which can provide the RT index of behavioral inhibitory control that the Go/No-go task does not have (Yuan et al., 2008, 2012).

\section{DATA AVAILABILITY STATEMENT}

The datasets generated for this study are available on request to the corresponding author.

\section{ETHICS STATEMENT}

The studies involving human participants were reviewed and approved by the Ethics Committee of Hunan Normal University. The patients/participants provided their written informed consent to participate in this study.

\section{AUTHOR CONTRIBUTIONS}

RX and JieC designed the study. RX, JieC, CL and JiejC wrote the manuscript and carried out all data analyses. RX, CL and JiejC conducted data collection. All authors contributed to and approved the final version of the manuscript.

\section{FUNDING}

This research was supported by the National Natural Science Foundation of China (NSFC31771240), Foundation of Education Department of Hunan Province of China (18A036), Hunan Provincial Fund for Philosophy and Social Sciences (15YBA263), and the 13th Five-years Plan of national education science the key Research topics of the Ministry of Education (DBA180316).

Cohen, J. (1988). Statistical Power Analysis for Behavioural Sciences. 2nd Edn. New York, NY: Academic Press.

Day, R.-F., Lin, C.-H., Huang, W.-H., and Chuang, S.-H. (2009). Effects of music tempo and task difficulty on multi-attribute decision-making: an eye-tracking approach. Comput. Hum. Behav. 25, 130-143. doi: 10.1016/j.chb.2008.08.001

Diamond, A. (2013). Executive functions. Annu. Rev. Psychol. 64, 135-168. doi: 10.1146/annurev-psych-113011-143750

Enriquez-Geppert, S., Konrad, C., Pantev, C., and Huster, R. J. (2010). Conflict and inhibition differentially affect the N200/P300 complex in a combined go/nogo and stop-signal task. NeuroImage 51, 877-887. doi: 10.1016/j.neuroimage.2010. 02.043

Falkenstein, M., Hoormann, J., and Hohnsbein, J. (1999). ERP components in Go/Nogo tasks and their relation to inhibition. Acta Psychol. 101, 267-291. doi: 10.1016/s0001-6918(99)00008-6 
Faul, F., Erdfelder, E., Lang, A. G., and Buchner, A. (2007). G*Power 3: a flexible statistical power analysis program for the social, behavioral, and biomedical sciences. Behav. Res. Methods 39, 175-191. doi: 10.3758/bf03193146

Gajewski, P. D., and Falkenstein, M. (2013). Effects of task complexity on ERP components in Go/Nogo tasks. Int. J. Psychophysiol. 87, 273-278. doi: 10.1016/j. ijpsycho.2012.08.007

Huster, R. J., Westerhausen, R., Pantev, C., and Konrad, C. (2010). The role of the cingulate cortex as neural generator of the N200 and P300 in a tactile response inhibition task. Hum. Brain Mapp. 31, 1260-1271. doi: 10.1002/hbm. 20933

Jodo, E., and Kayama, Y. (1992). Relation of a negative ERP component to response inhibition in a Go/Nogo task. Electroencephalogr. Clin. Neurophysiol. 82, 477-482. doi: 10.1016/0013-4694(92)90054-1

Karageorghis, C. I., Jones, L., Priest, D.-L., Akers, R. I., Clarke, A., Perry, J. M., et al. (2011). Revisiting the relationship between exercise heart rate and music tempo preference. Res. Q. Exerc. Sport 82, 274-284. doi: 10.1080/02701367. 2011.10599755

Kim, J., Strohbach, C. A., and Wedell, D. H. (2018). Effects of manipulating the tempo of popular songs on behavioral and physiological responses. Psychol. Music 47, 392-406. doi: 10.1177/0305735618754688

Levitin, D. J., Grahn, J. A., and London, J. (2018). The psychology of music: rhythm and movement. Annu. Rev. Psychol. 69, 51-75. doi: 10.1146/annurev-psych122216-011740

Luijten, M., Littel, M., and Franken, I. H. (2011). Defcits in inhibitory control in smokers during a Go/NoGo task: an investigation using eventrelated brain potentials. PLoS One 6:e18898. doi: 10.1371/journal.pone.0018898

McElrea, H., and Standing, L. (1992). Fast music causes fast drinking. Percept. Mot. Skills 75:362. doi: 10.2466/pms.1992.75.2.362

Milliman, R. E. (1982). Using background music to affect the behavior of supermarket shoppers. J. Marketing 46, 86-91. doi: 10.2307/1251706

Milliman, R. E. (1986). The influence of background music on the behavior of restaurant patrons. J. Consum. Res. 13, 286-289. doi: 10.1086/ 209068

Navarro, J., Osiurak, F., and Reynaud, E. (2018). Does the tempo of music impact human behavior behind the wheel? Hum. Factors 60, 556-574. doi: 10.1177/0018720818760901
Nieuwenhuis, S., Yeung, N., van den Wildenberg, W., and Ridderinkhof, K. R. (2003). Electrophysiological correlates of anterior cingulate function in a go/no-go task: effects of response conflict and trial type frequency. Cogn. Affect. Behav. Neurosci. 3, 17-26. doi: 10.3758/cabn.3.1.17

North, A. C., Hargreaves, D. J., and Heath, S. J. (1998). Musical tempo and time perception in a gymnasium. Psychol. Music 26, 78-88. doi: 10.1177/0305735698261007

Sutton, C. J. C., and Lowis, M. J. (2008). The effect of musical mode on verbal and spatial task performance. Creat. Res. J. 20, 420-426. doi: 10.1080/10400410802391884

Van Veen, V., and Carter, C. S. (2002). The timing of action-monitoring processes in the anterior cingulate cortex. J. Cogn. Neurosci. 14, 593-602. doi: 10.1162/08989290260045837

Yuan, J. J., He, Y. Y., Zhang, Q. L., Chen, A. T., and Li, H. (2008). Gender differences in behavioral inhibitory control: ERP evidence from a two-choice oddball task. Psychophysiology 45, 986-993. doi: 10.1111/j.1469-8986.2008. 00693.x

Yuan, J. J., Meng, X. X., Yang, J. M., Yao, G. H., Hu, L., and Yuan, H. (2012). The valence strength of unpleasant emotionmodulates brain processing of behavioral inhibitory control: neural correlates. Biol. Psychol. 89, 240-251. doi: 10.1016/j.biopsycho.2011.10.015

Zuk, J., Benjamin, C., Kenyon, A., and Gaab, N. (2014). Behavioral and neural correlates of executive functioning in musicians and non-musicians. PLoS One 9:e99868. doi: 10.1371/journal.pone.0099868

Conflict of Interest: The authors declare that the research was conducted in the absence of any commercial or financial relationships that could be construed as a potential conflict of interest.

Copyright (c) 2020 Xiao, Liu, Chen and Chen. This is an open-access article distributed under the terms of the Creative Commons Attribution License (CC BY). The use, distribution or reproduction in other forums is permitted, provided the original author(s) and the copyright owner(s) are credited and that the original publication in this journal is cited, in accordance with accepted academic practice. No use, distribution or reproduction is permitted which does not comply with these terms. 